\title{
Potential Compressive Sites of the Anterior Interosseous Nerve in the Proximal Forearm: An Anatomic Study ${ }^{*}$
}

\author{
Laurent Mathieu $^{1}$, Eric R. Simms ${ }^{2}$, Frédéric Rongiéras ${ }^{1}$, Eric J. Voiglio ${ }^{3}$, Christophe Oberlin ${ }^{4}$ \\ ${ }^{1}$ Desgenettes Military Academic Hospital, Lyon, France; ${ }^{2}$ Tulane University School of Medicine, New Orleans, USA; ${ }^{3}$ Centre Hos- \\ pitalier Lyon-Sud, University, Lyon, France; ${ }^{4}$ Saints-Pères University Center, Paris Descartes University, Paris, France. \\ Email: laurent_tom2@yahoo.fr
}

Received September $26^{\text {th }}, 2012$; revised October $26^{\text {th }}, 2012$; accepted November $16^{\text {th }}, 2012$

\begin{abstract}
Background: The etiology and treatment of spontaneous paralysis variants of anterior interosseous nerve (AIN) syndrome remains controversial. Variation and multiple sites of potential compression complicate the successful performance of neurolysis. This anatomic study of the AIN and sites of potential compression in the proximal forearm facilitates critical steps involved in neurolytic procedures and management. Methods: Upper extremities of twelve cadavers were examined to evaluate potential sites of AIN compression in the proximal forearm. Potential sites of musculoaponeurotic compression were evaluated, including: lacertus fibrosus; inferior fibrous arch of the humeral head of the pronator teres (PT) muscle; inferior fibrous arch of the ulnar head of the PT muscle; fibrous arch in the flexor digitorum superficialis (FDS) muscle; Gantzer's muscle; and vascular structures near the AIN and median nerve. Results: The AIN arose at a mean distance of $54.5 \mathrm{~mm}$ distal to the elbow from the posterior $(n=9)$ or ulnar side $(n=3)$ of the median nerve. Relative positions of AIN branches were variable. A fibrous arch was found between the lacertus fibrosus and the PT in two cases. Nine cadavers had two fibrous arches in the PT and FDS, and three cadavers had one arch. An accessory head in the FDS was found to be a risk of AIN compression. Gantzer's muscle was present in six cases, crossing the AIN superficially. Two potentially compressive vascular arches were identified. Conclusions: Our observations confirm that multiple musculoaponeurotic and/or vascular structures can contribute to AIN compression in the proximal forearm. Understanding the complex anatomic relationships of this nerve is crucial to improving outcomes of neurolysis in cases of non-regressive AIN paralysis.
\end{abstract}

Keywords: Anterior Interosseous Nerve; Anatomy; Compression; Paralysis; Neurolysis

\section{Introduction}

Anterior interosseous nerve syndrome (AINS) can present as two distinct entities: a post-traumatic or iatrogenic paralysis which can concern the orthopedic surgeon, and a spontaneous paralysis. Posttraumatic injuries of the AIN are generally caused by forearm fractures or elbow dislocations [1-3]. Iatrogenic injuries may result from open surgery [4,5], elbow or shoulder arthroscopy [6,7], but also from slings [8] or veinepuncture [9]. Spontaneous paralysis of the AIN has described as a neurotic phenomenon $[10,11]$, but was recently attributed to the existence of musculoaponeurotic structural compressions [12,13] or Hourglass-shaped compressions of nerve fasciculae [14-16].

Due to the relative rarity of this syndrome, few controlled studies exist to determine the most effective treatment techniques [17]. Most common treatment strategies for posttraumatic or spontaneous AIN paralysis

*The authors declare they have no conflict of interest. involve starting with conservative management. However surgical decompression is warranted if there are no signs of recovery two or three months after the onset of paralysis $[17,18]$. The deep localization of the AIN, anatomic variation and multiple sites of potential compression complicate the successful performance of neurolysis. There is a paucity of recent literature describing the potential compressive sites of the AIN. Our anatomic study of the potential sites of AIN compression at the proximal forearm fills a void in the literature, to facilitate the critical identification steps involved in neurolytic procedures and management.

\section{Materials and Methods}

A Cadaveric study to evaluate potential sites of AIN compression in the proximal forearm was performed in the Department of Anatomy, Saints-Pères University Center, Paris Descartes University, France. The upper extremities of twelve fresh cadavers were dissected and examined. Six male and six female cadavers without 
known history of upper extremity trauma were randomly selected. Mean age at the time of death was 82 years (range 62 - 84 years). Only one upper extremity was dissected on each cadaver: seven right sided cadaveric upper extremities and five left sided.

Dissections exposed the full course of the AIN in the proximal forearm, as well as the course of the median nerve, extending from the antecubital fossa to the emergence of the AIN. The potential sites of musculoaponeurotic compression as identified by Dellon and Mackinnon [19] were evaluated. These included: 1) the lacertus fibrosus (LF); 2) the fibrous arch inferior to the humeral head of the pronator teres (PT) muscle; 3) the fibrous arch inferior to the ulnar head of the PT muscle; 4) the fibrous arch within the flexor digitorum superficialis (FDS) muscle; 5) the accessory head of the flexor pollicis longus (FPL) muscle, also known as Gantzer's muscle. The topographic relationships of Gantzer's muscle to the AIN were evaluated using the system described by $\mathrm{Oh}$ et al. [20]. Vascular structures near the AIN and median nerve were likewise analyzed, and representative anatomy was photographed. Topographic measurements between the specified anatomic structures and surrounding superficial osseous landmarks were obtained using calipers. The biepicondylar line of the humerus was used as a reference point. The measurements taken included the distance ("D") from the biepicondylar line to each musculoaponeurotic, vascular, and neural structure. For the AIN and its branches, distances were measured from nerve origin on the main nerve. For musculoaponeurotic and vascular structures, distances were measured from the point crossing the AIN or median nerve. Forearm length was measured from the biepicondylar line of the humerus to the ulnar styloid process. Data from the present study were compared to that of previously published studies $[19,21,22]$.

\section{Results}

\subsection{Biometry of the AIN}

The AIN was found originated from the posterior aspect of the median nerve in nine specimens, and from the ulnar side of the median nerve in three specimens. Origins arose at a mean distance of $54.5 \mathrm{~mm}$ (range 41 - $76 \mathrm{~mm}$; Table 1) distal to the biepicondylar line of the humerus. These origins were localized beneath the humeral head of the PT in nine specimens, proximal to the PT in two specimens, and distal to the PT in one specimen. The relative positions of AIN branches were variable (Table 1). AIN branches leading to the FPL muscle arose distally to those leading to the flexor digitorum profundus (FDP) muscles in nine specimens. Branches leading to the second and third flexor digitorum profundus muscles (FDP2 and FDP3) had a common origin off of the AIN in six specimens. An inconstant branch of the AIN to the FDS muscle was present in two specimens. A MartinGruber communicating branch was the most proximal branch of the AIN in two specimens (Figure 1).

\subsection{Musculoaponeurotic Arches}

In all specimens, the LF was present under the fascia of

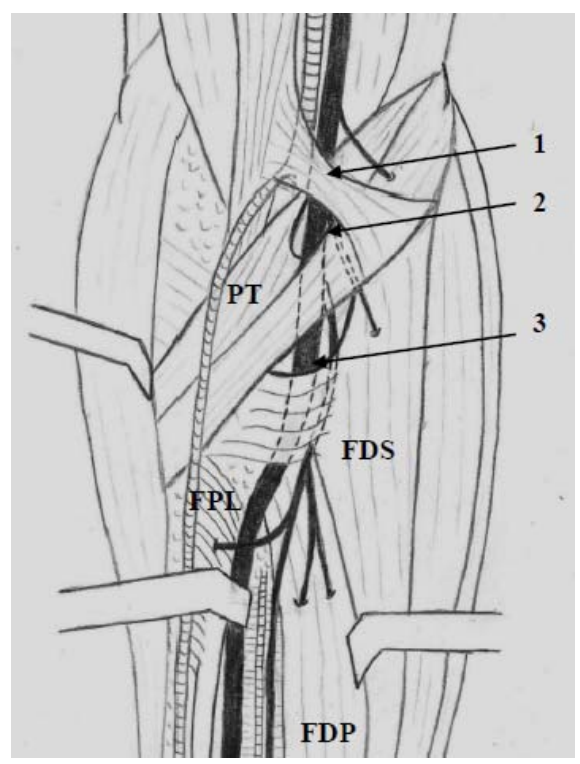

Figure 1. Illustration of the AIN in the proximal forearm: 1) LF; 2) arch of the PT muscle; 3) arch of the FDS muscle.

Table 1. Measurements involving branches of the AIN (distance D in millimeters).

\begin{tabular}{|c|c|c|c|c|c|c|c|c|c|c|c|c|}
\hline Specimen & 1 & 2 & 3 & 4 & 5 & 6 & 7 & 8 & 9 & 10 & 11 & 12 \\
\hline Forearm lengh & 215 & 230 & 360 & 265 & 245 & 250 & 260 & 195 & 245 & 260 & 220 & 270 \\
\hline Origin of the AIN & 44 & 55 & 55 & 43 & 41 & 76 & 63 & 50 & 54 & 64 & 54 & 55 \\
\hline Branch to the FDS & - & - & - & - & - & - & - & - & - & 75 & 63 & - \\
\hline Branch to the ulnar nerve & 66 & - & - & - & - & - & - & - & 80 & - & - & - \\
\hline Branch to the FDP2 & 75 & 71 & 98 & 81 & 82 & 92 & 82 & 65 & 84 & 89 & 71 & 78 \\
\hline Branch to the FDP3 & 70 & 71 & 95 & 69 & 82 & 92 & 82 & 65 & 84 & 93 & 64 & 80 \\
\hline Branch to the FPL & 77 & 70 & 101 & 73 & 96 & 87 & 91 & 83 & 115 & 103 & 74 & 88 \\
\hline
\end{tabular}


the humeral head of the PT. The LF crossed the median nerve at a mean distance of $25.1 \mathrm{~mm}$ (range 17 - $35 \mathrm{~mm}$; Table 2) from the biepicondylar line of the humerus. Two specimens demonstrated a conjoined fibrous arch formed by the LF and PT fascias, and this arch was in a position which could cause potential compression of the median nerve across the antecubital fossa (Figure 2).

The PT muscles examined exhibited only one head (humeral) in four specimens, and two heads (humeral and ulnar) in eight specimens. In the cadavers with two PT muscle heads, a fibrous arch was present between both heads. In cadavers with a single humeral head present, a fibrous arch was noted in only two of the four specimens. The mean distance from the biepicondylar line to the fibrous arch of the PT muscle was $65.2 \mathrm{~mm}$ (range 44 $84 \mathrm{~mm}$; Table 2).

The FDS muscle showed great anatomic variation in the cadavers examined. Three heads were present in eight specimens: a humero-ulnar head from the medial epicondyle and coronoid process, a radial head from the anterior radial crest, and an accessory head from the interosseous membrane located very close to the AIN and median nerve.Three specimens had FDS muscles with two heads (humero-ulnar and radial), and one specimen had a single humero-ulnar head.

A fibrous arch was found in the eleven specimens which had either two or three heads of their FDS muscles. The mean distance from the biepicondylar line to the fibrous arch of the FDS muscle was $73.8 \mathrm{~mm}$ (range 55 $90 \mathrm{~mm}$; Table 2). In one specimen an additional fibrous band was identified between the humeral head of the PT muscle and the fibrous arch of the FDS muscle.

On the whole, the number of fibrous arches crossing the AIN from the PT and FDS muscles was variable (Table 3). Nine specimens demonstrated two arches, and six of these nine cadavers had an anatomic arrangement capble of nerve compression, recognized as a tight arch in the FDS muscle due to the presence of an accessory head. Gantzer's muscle was present in six of the twelve cadavers. In all six of these specimens, Gantzer's muscle originated from the flexor pronator mass, passed anterior or lateral to the AIN, and inserted into the FPL tendon (Figure 3). Depending on shape, Gantzer's muscle was classified into one of three categories: "voluminous," "fusiform," or "strap-like” (slender). Two muscles were classified as voluminous, two as fusiform, and two as strap-like. The AIN crossed the muscular part of Gan-

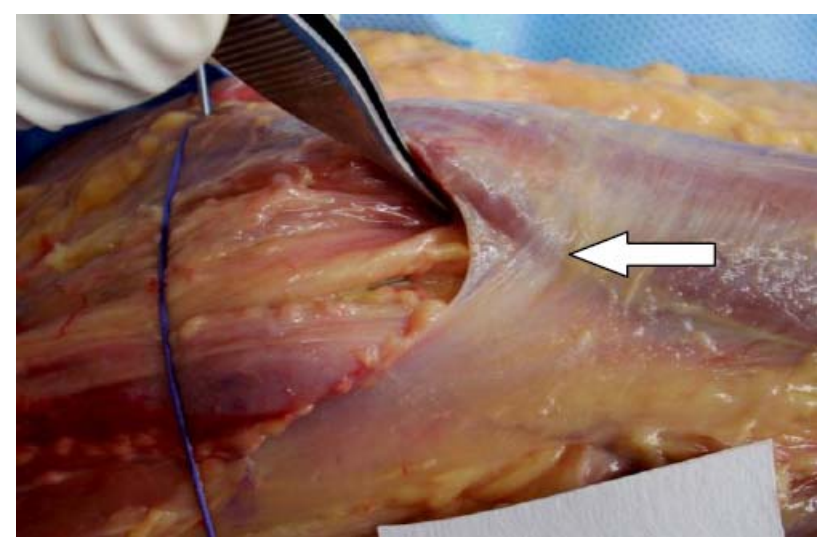

Figure 2. Fibrous arch formed by the LF and the PT fascia.

Table 2. Measurements involving musculoaponeurotic structures (distance D in millimeters).

\begin{tabular}{|c|c|c|c|c|c|c|c|c|c|c|c|c|}
\hline Specimen & 1 & 2 & 3 & 4 & 5 & 6 & 7 & 8 & 9 & 10 & 11 & 12 \\
\hline Forearm lengh & 215 & 230 & 360 & 265 & 245 & 250 & 260 & 195 & 245 & 260 & 220 & 270 \\
\hline Lacertus fibrosus & 20 & 35 & 34 & 27 & 27 & 19 & 34 & 17 & 27 & 20 & 20 & 22 \\
\hline Arch of the PT & 45 & - & 56 & 80 & 44 & 56 & 71 & 84 & 67 & 83 & - & 66 \\
\hline Arch of the FDS & 55 & 55 & 74 & 83 & 76 & 58 & - & 84 & 73 & 90 & 78 & 86 \\
\hline Gantzer's muscle & 66 & 118 & 116 & - & 88 & 97 & - & - & - & - & 74 & - \\
\hline
\end{tabular}

Table 3. Arches formed by the PT and FDS muscles.

\begin{tabular}{cccc}
\hline & No PT arch & $\begin{array}{c}\text { Arch under humeral } \\
\text { head of PT }\end{array}$ & $\begin{array}{c}\text { Arch between humeral and } \\
\text { ulnar heads of PT }\end{array}$ \\
\hline No FDS arch & 0 & 0 & 1 \\
Arch under humero-ulnar head of FDS & 1 & 1 & 1 \\
Arch between humero-ulnar and accessory heads of FDS & 1 & $\mathbf{3}$ & $\mathbf{2}$ \\
Totals & $\mathbf{2}$ & $\mathbf{8}$ \\
\hline
\end{tabular}




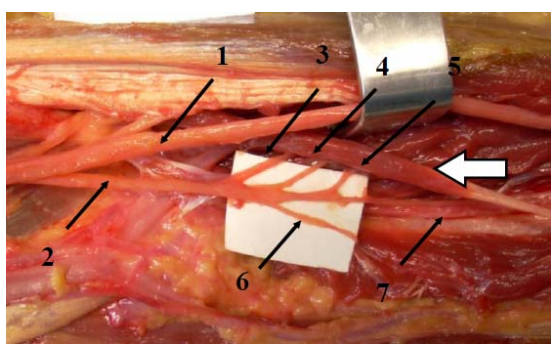

Figure 3. Gantzer's muscle (white arrow) and branches of the AIN: 1) median nerve; 2) AIN; 3) Martin-Gruber anastomosis; 4) branch leading to the FDP2; 5) branch leading to the FDP3; 6) branch leading to the FPL; 7) branch leading to the pronator quadratus.

tzer's muscle in four specimens (type A) according to Oh et al. [20]); crossed the tendinous part of Gantzer's muscle in one specimen (type B); and coursed lateral to Gantzer's muscle in one specimen (type $\mathrm{C}$ ).

\subsection{Vascular Arches}

Vascular structures may be at risk for causing nerve compression when they cross the nerve in tight anatomic tunnels. This constrained relationship between artery and nerve occurred in eight specimens in the present study, two of which demonstrated potentially compressive vascular arches. A collateral branch of the ulnar artery going to the FDS muscle crossed the AIN superficially inferior to the FDS fascial arch in all eight of these specimens. Five of these also exhibited collateral branches of the brachial artery going to the PT muscle which crossed the median nerve superficially at the level of the LF.

\section{Discussion}

The goal of this anatomic study was to provide a basis for the crucial identification steps of neurolysis of the AIN, to increase surgical precision and decrease potential morbidity. Despite a limited number of cadaveric specimens, our study demonstrates the main anatomic variants of the AIN and surrounding musculoaponeurotic strucatures. In addition to anatomic complexity, spatial relationships of the AIN in the proximal forearm change with pronosupination. Therefore, another significant limitation of this study is the technical difficulty in performing dynamic evaluations of potential nerve compression sites using a cadaveric model.

\subsection{Anatomic Variations of the AIN}

Tubbs et al. [21] performed a series examining ten cadaveric upper extremities and noted that the AIN originated at a mean distance of $5.4 \mathrm{~cm}$ from the medial epicondyle of the humerus. Subsequently, Canovas et al. [22] reported a more proximal origin of the AIN, with a mean distance of $4.3 \mathrm{~cm}$ from the biepicondylar line of the el- bow. Multiple authors have noted that the AIN originates from the median nerve at the level of the PT muscle or more distal $[23,24]$. The present study demonstrated that the AIN originated inferior to the PT muscle in nine specimens, proximal to the PT in two specimens, and more distal in only one specimen. There are likewise variable anatomic descriptions in the literature, some authors asserting that the AIN originates from the radial or posterior side of the median nerve $[19,23,24]$, while others note the AIN arising from the ulnar or posterior side of the median nerve [20]. Dellon and Mackinnon [19] state that a radial origin of the AIN inferior to a fibrous arch represents an anatomic arrangement at risk of nerve compression. In the present study, the AIN never originated from the radial side of the median nerve. In their biometric study, Canovas et al. [22] found that AIN branches leading to the FDP originated commonly from the AIN in all of the cadavers they used, with a mean distance of $5.9 \mathrm{~cm}$ from the biepicondylar line of the elbow, $1.75 \mathrm{~cm}$ distal to the origin of the AIN. They also noted a proximal branch leading to the FDS muscle in two cases. They nonetheless concluded that the level of origin of the AIN and its branches is less variable than the level of origin of most other muscular nerve branches.

\subsection{Potential Sites of Compression}

Dellon and Mackinnon [19] performed a series on 31 cadaveric arms to review anatomic structures which may cause AIN or median nerve compression in the forearm. They discovered a musculoaponeurotic network formed by the multiple heads of the PT and FDS muscles arranged in a variable number of arches across these nerves. Only a single specimen showed no arches, while twenty specimens demonstrated one arch each, and another ten had two arches each. A similar analysis was made in the present study; however the greater part of the specimens dissected demonstrated two fibrous arches. Fibrous arches were present in all PT muscles with two heads, and in 50\% of PT muscles with a single head. Johnson et al. [25] demonstrated that the humeral head of the PT muscle contained a fibrous arch in $40 \%$ of the 40 cadavers which they dissected. When present, this fibrous component extended into the ulnar head. A fibrous arch was likewise identified in all FDS muscles with two or three heads. In the present study, we noted that the presence of an accessory head of the FDS muscle represents an anatomic arrangement at risk for nerve compression because the fibrous arch crosses the median nerve and the origin of the AIN in a very tight configuration with this presentation.

AIN compression in the forearm can occur proximal to its origin on the median nerve, inferior to the LF [13]. Dellon and Mackinnon [19] furthermore showed that a superficial head of the PT arising proximal to the medial 
humeral epicondyle can become contiguous with the LF, and may therefore cause compression of the median nerve at the antecubital fossa. In the current study this anatomic arrangement was found in only three specimens.

The overall incidence of presence of the Gantzer's muscle observed in this study was 50\%. A number of authors have reported variable incidence of this muscle [26]. Bergman et al. [27] reported an incidence ranging from 33.3\% in European Caucasians to $89.3 \%$ in people of African descent. The most frequent site of origin of Gantzer's muscle is reported to be the medial epicondyle of humerus $[25,28]$, however other variants are recognized to originate from the coronoid process or the FDS muscle [20]. Gantzer's muscle observed in our study complimented the observations of Pai et al. [26] and Mangini, [28] as we likewise noted its position between the median nerve anteriorly and the AIN posteriorly. By way of contrast, Dellon and Mackinnon [19] stated that Gantzer's muscle always lies posterior to the median nerve or AIN. Mahakkanukrauh et al. [29] found that the AIN more commonly crosses Gantzer's muscle laterally, a configuration that we noted in a single specimen. The topographic classification of the relationship between the AIN and Gantzer's muscle proposed by Oh et al. [20] is considered helpful in understanding the mechanism of AIN compression by this muscle: This compressive phenolmenon more commonly occurs in types A and B, especially when the nerve makes contact with "strap-like" muscle morphology [20].

\subsection{Clinical and Surgical Applications}

Because of anatomic variation of AIN branches, dissociated paralyses of the FDP2 and FPL muscles have been reported [30,31]. In the Werner's series [32] of 69 patients, both of these muscles were paralyzed in 34 patients, only the FPL muscle in 25, and only the FDP2 muscle in ten. In these cases of individual muscular paralysis, the diagnosis of AINS is more difficult to make. Duteille et al. [31] highlight the example that a posttraumatic isolated paralysis of the FPL muscle may be mistaken for tendinous rupture. The multiple potential compressive structures in the proximal forearm explain why electromyographic studies sometimes fail to localize sites of AIN compression. Dellon and Mackinnon [19] thought that multiple compressive sites may be involved, each of which would be insufficient to be detected by electromyography (EMG), but whose combined effect could result in overall dysfunction of the nerve. Therefore, in cases of non-regressive paralysis of the AIN, neurolysis remains indicated despite the absence of a single well-identified compressive site via EMG.

When a surgical release of the AIN is performed, the LF and any fibrous arch contiguous with the PT muscle should be excised. The median nerve must be dissected on the radial side to avoid iatrogenic injury to the AIN and other motor branches arising from the ulnar or posterior sides. All fibrous arches crossing over the AIN at the level of the PT and FDS muscles will likewise have to be released for complete relief of compressive symptoms. The ulnar head of the PT muscle can be divided without functional loss, and the surgeon's finger should be advanced through the pronator arch to identify the presence or absence of a second arch in the FDS muscle [19]. Gantzer's muscle, easily identified in the middle of the forearm, has to be excised. The two vascular arches crossing over the median nerve and the AIN should be cauterized and divided as they likely contribute to nerve compression. When there are no constriction bands or muscular compressions noted on the nerve, Omura et al. [33] suggest that careful microscopic surgical exploration should be performed to evaluate for hourglass-like constrictions causing interfascicular neurolysis.

\section{Conclusion}

Multiple musculoaponeurotic and/or vascular structures can contribute to AIN compression in the proximal forearm. Understanding the complex anatomic relationships of this nerve is crucial to improving outcomes of neuronlysis in cases of non-regressive AIN paralysis. However, proximal compression of the median nerve may selectively damage fasciculae destined for the AIN, mimicking a more distal AIN lesions. Additional studies are warranted to identify proximal entrapment sites that may be involved in AINS.

\section{REFERENCES}

[1] A. J. Arenas, F. J. Artazcoz, A. Tejero and C. Arias, “Anterior Interosseous Nerve Injury Associated with a Monteggia Fracture-Dislocation,” The Acta Orthopaedica Belgica, Vol. 67, No. 1, 2001, pp. 77-80.

[2] D. J. Bamford and D. Stanley, "Anterior Interosseous Nerve Paralysis: An underdiagnosed Complication of Supracondylar Fracture of the Humerus in Children,” Injury, Vol. 20, No. 5, 1989, pp. 294-312.

[3] Y. Mirovsky, D. Hendel and N. Halperin, “Anterior Interosseus Nerve Palsy Following Closed Fracture of the Proximal Ulna," Archives of Orthopaedic and Traumatic Surgery, Vol. 107, No. 1, 1988, pp. 61-64. doi:10.1007/BF00463528

[4] P. Keogh, H. Khan, E. Cooke and G. McCoy, "Loss of Flexor Pollicis Longus Function after Plating of the Radius: Report of Six Cases,” The Journal of Hand Surgery, Vol. 22B, No. 3, 1997, pp. 375-376. doi:10.1016/S0266-7681(97)80405-6

[5] L. I. Katolik and M. S. Cohen, "Anterior Interosseous Nerve Palsy after Open Capsular Release for Elbow Stiffness: Report of Two Cases," The Journal of Hand Surgery, Vol. 34, No. 2, 2009, pp. 288-291.

[6] E. W. Kelly, B. F. Morrey and S. W. O’Driscoll, “Com- 
plications of Elbow Arthroscopy,” Journal of Bone \& Joint Surgery, Vol. 83, No. 1, 2001, pp. 25-34.

[7] M. Sisco and G. A. Dumanian, “Anterior Interosseous Nerve Syndrome Following Shoulder Arthroscopy,” Journal of Bone \& Joint Surgery, Vol. 89, No. 2, 2007, pp. 392-395. doi:10.2106/JBJS.F.00445

[8] D. B. O’Neill, B. Zarins, R. H. Gelberman, T. M. Keating and D. Louis, "Compression of the Anterior Interosseous Nerve after Use of a Sling for Dislocation of the Acromioclavicular Joint: A Report of Two Cases,” Journal of Bone \& Joint Surgery, Vol. 72, No. 7, 1990, pp. 11001102.

[9] M. E. Puhaindran and H. P. Wong, “A case of Anterior Interosseous Nerve Syndrome after Peripherally Inserted Central Catheter (PICC) Line Insertion,” Singapore Medical Journal, Vol. 44, No. 12, 2003, pp. 653-655.

[10] M. J. Parsonage and J. W. A. Turner, "Neuralgic amyotrophy: The Shoulder-Girdle Syndrome," Lancet, Vol. 251, No. 6513, 1948, pp. 973-978. doi:10.1016/S0140-6736(48)90611-4

[11] L. G. Kiloh and S. Nevin, "Isolated Neuritis of the Anterior Interosseous Nerve,” British medical journal, Vol. 1, No. 4763, 1952, pp. 850-851.

[12] C. B. Fearn and J. W. Goodfellow, "Anterior Interosseous Nerve Palsy,” Journal of Bone \& Joint Surgery, Vol. 47B, No. 1, 1965, pp.91-93.

[13] E. B. Kaplan, M. Spinner, "The Anterior Interosseous Nerve Syndrome,” Journal of Bone \& Joint Surgery, Vol. 50, No. 3, 1968, pp. 521-523.

[14] H. M. Englert, "Partielle Faszikuläre Medianus-Atrophie Uneklärter Genese,” Handchirurgie, Vol. 8, 1976, pp. 61 $-62$.

[15] P. Haussmann and K. Kendel, "Oligofaszikulâres Medianus-Compressions Syndrome,” Handchirurgie, Vol. 13, 1981, pp. 268-271.

[16] A. Nagano, K. Shibata, H. Tokimura, S. Yamamoto and Y. Tajiri, "Spontaneous Anterior Interosseous Nerve Palsy with Hourglass-Like Fascicular Constriction within the Main Trunk of the Median Nerve," The Journal of Hand Surgery, Vol. 21A, No. 2, 1996, pp. 266-270.

[17] D. Ulrich, A. Piatkowski and N. Pallua, “Anterior Interosseous Nerve Syndrome: Retrospective Analysis of 14 Patients," Archives of Orthopaedic and Traumatic Surgery, Vol. 131, No. 11, 2011, pp. 1561-1565. doi:10.1007/s00402-011-1322-5

[18] M. K. Sood and F. D. Burke, "Anterior Interosseous Nerve Palsy," The Journal of Hand Surgery, Vol. 22, No. 1, 1997, pp. 64-68. doi:10.1016/S0266-7681(97)80020-4

[19] A. L. Dellon and S. E. Mackinnon, "Musculoaponeurotic Variations along the Course of the Median Nerve in the Proximal Forearm," The Journal of Hand Surgery, Vol. 12, No. 3, 1987, pp. 359-363. doi:10.1016/0266-7681(87)90189-6

[20] C. S. Oh, I. H. Chung and K. S. Koh, “Anatomical Study of the Accessory Head of the Flexor Pollicis Longus and the Anterior Interosseous Nerve in Asians," Clinical Anatomy, Vol. 13, No. 6, 2000, pp. 434-438.
[21] R. S. Tubbs, J. W. Custis, E. G. Salter, J. C. Wellons, J. P. Blount and W. J. Oakes, "Quantification of and Superficial Surgical Landmarks for the Anterior Interosseous Nerve,” Journal of Neurosurgery, Vol. 104, No. 5, 2006, pp. 787-791. doi:10.3171/jns.2006.104.5.787

[22] F. Canovas, P. Mouilleron and F. Bonnel, "Biometry of the Muscular Branches of the Median Nerve to the Forearm,” Clinical Anatomy, Vol. 11, No. 4, 1998, pp. 239245.

[23] P. Seror, “Anterior Interosseous Nerve Lesions: Clinical and Electrophysiological Features,” Journal of Bone \& Joint Surgery, Vol. 78B, No. 2, 1996, pp. 238-241.

[24] D. H. Kim, J. A. Murovic, Y. Y. Kim and D. G. Kline, "Surgical Treatment and Outcomes in 15 Patients with Anterior Interosseous Nerve Entrapments and Injuries," Journal of Neurosurgery, Vol. 104, No. 5, 2006, pp. 757765. doi:10.3171/jns.2006.104.5.757

[25] R. K. Johnson, M. Spinner and M. M. Shrews, "Median Nerve Entrapment in the Proximal Forearm,” The Journal of Hand Surgery, Vol. 19, No. 1, 1979, pp. 48-51.

[26] M. M. Pai, S. R. Nayak, A. Krishnamurthy, R. Vadgaonkar, L. V. Prabhu, A. V. Ranade, J. P. Janardhan and R. Rai, "The Accessory Head of Flexor Pollicis Longus and Flexor Digitorum Profundus: Incidence and Morphology," Clinical Anatomy, Vol. 21, No. 3, 2008, pp. 252258. doi:10.1002/ca.20612

[27] R. A. Bergman, A. K. Afifi and R. Mlyauchi, "Illustrated Encyclopedia of Human Anatomic Variation, Opus I: Muscular System: Alphabetical Listing of Muscles: F,” 2007. www.anatomyatlases.org/AnatomicVariants/MuscularSys tem/Text/F/22Flexor.shtml

[28] U. Mangini, "Flexor Pollicis Longus Muscle: Its Morphology and Clinical Significance,” Journal of Bone \& Joint Surgery, Vol. 42, No. 3, 1960, pp. 467-470.

[29] P. Mahakkanukrauh, P. Surin, N. Ongkana, M. Sethadavit, P. Vaidhayakarn, "Prevalence of Accessory Head of Flexor Pollicis Longus Muscle and Its Reltion to Anterior Interosseous Nerve in Thai Population," Clinical Anatomy, Vol. 17, No. 8, 2004, pp.631-635. doi:10.1002/ca.20016

[30] A. Nagano, "Spontaneous Anterior Interosseous Nerve Palsy,” Journal of Bone \& Joint Surgery, Vol. 85B, No. 3, 2003, pp. 313-318. doi:10.1302/0301-620X.85B3.14147

[31] F. Duteille, B. Amara, G. Dautel and M. Merle, "Atteinte Isolée du Long Fléchisseur du Pouce Dans le Syndrome Du Nerf Inter-Osseux Antérieur," Revue de Chirurgie Orthopedique, Vol. 86, 2000, pp. 306-309.

[32] C. O. Werner, "The Anterior Interosseous Nerve Syndrome,” International Orthopaedics, Vol. 13, No. 3, 1989, pp. 193-197. doi:10.1007/BF00268047

[33] T. Omura, A. Nagano, H. Murata, M. Takahashi, H. Ogihara and K. Omura, "Simultaneous Anterior and Posterior Interosseous Nerve Paralysis with Several Hourglass-Like Fascicular Constrictions in the Both Nerves,” The Journal of Hand Surgery, Vol. 26A, No. 6, 2001, pp. 1088-1092. 